\title{
Chemical Safety Concerns of Sulfur Mustard Analog, 2-Chloroethyl Ethyl Sulfide, in Laboratory Study
}

\author{
Feng Ye (iD) ${ }^{1,}$, Yan Sai (iD ${ }^{1}$ and Zhongmin Zou (iD ${ }^{1, * *}$ \\ ${ }^{1}$ Department of Chemical Defense Medicine, Army Medical University, Third Military Medical University, Chongqing, P.R. China \\ "Corresponding author: Department of Chemical Defense Medicine, Army Medical University, Third Military Medical University, Chongqing, P.R. China. Email: \\ 293312620@qq.com \\ Corresponding author: Department of Chemical Defense Medicine, Army Medical University, Third Military Medical University, Chongqing, P.R. China. Email: \\ zmzou@tmmu.edu.cn
}

Received 2021 November 20; Accepted 2021 December 26.

\begin{abstract}
Sulfur mustard (SM), a classic chemical weapon in the vesicant category, can induce severe damage, for which the therapy is still limited even today. Laboratory work is essential in unveiling toxicological effects and developing medical countermeasures. Sulfur mustard analog 2-chloroethyl ethyl sulfide (CEES), is employed in the lab for less toxicity. However, due to its similar characteristics to SM (being oily, hydrophobic, and volatile), the manipulation of CEES still needs special attention to avoid personnel injury and laboratory pollution. Here, to clear the chemical safety concerns in the laboratory study of CEES, the working procedure and experimental data are summarized, which might help educate new researchers to be skilled and professional.
\end{abstract}

Keywords: CEES, Vapor, Erosive, Laboratory Study, Chemical Safety

\section{Background}

Toxicology research is employed to investigate the toxic effects, underline mechanisms, and medical care on hazardous materials, commonly chemicals. Much experimental work should be taken in the medical or life science laboratory. Before running the study, the researcher needs to be first educated about chemical safety, especially in handling a novel or highly toxic substance. Sulfur mus$\operatorname{tard}(\mathrm{SM})$ is a synthetic vesicant agent that dates back to the early 19th century. It always imposes dangers to communities and individuals in public places and laboratories (14). Therefore, a less toxic SM analog, namely 2-chloroethyl ethyl sulfide (CEES), is employed to explore toxicological mechanisms and medical therapy for the category of vesicants (5-8).

CEES has a chemical structure similar to SM. Both are oily, hydrophobic, erosive, and quite different from many other skin-burning chemicals. Besides, they can be released into indoor air as a liquid spray (aerosol) or vapor. At room temperature, they are primarily a liquid hazard with low evaporation. With temperature increase, the vapor hazard increases. Although they are heavier than water, tiny droplets could still form a floating oil film on water surfaces while the droplets precipitate to the bottom and present a more prolonged hazard in contaminated areas (9). Although the vesicant characteristics have been well known, information about chemical safety in laboratory studies is still limited.

\section{Objectives}

This article summarizes the working procedure and experimental data to provide recommendations for other researchers to avoid occupational exposure to CEES in the laboratory study.

\section{Methods}

\subsection{Sub-packaging and Sealing}

CEES obtained commercially from Sigma (CAS number: 693-07-2, St. Louis, MO, USA) was about 5 or $25 \mathrm{~mL}$ in a brown glass bottle. Due to the small consumption in regular experiments, sub-packaging is necessary for easy and safe use. Noticeably, all procedures for handling CEES in the laboratory study should be taken in a qualified fume hood.

First, about 50 - $200 \mu \mathrm{L}$ CEES was transferred into a 0.5 mL Polypropylene EP tube (Sigma, USA) and further stored 
in a refrigerator at $4^{\circ} \mathrm{C}$. The lid of the CEES containment (both glass bottle and EP tube) should be sealed by sealing film to avoid leakage. If not sealed, CEES could evaporate slowly even at $4^{\circ} \mathrm{C}$, leaking and polluting the laboratory. Since the CEES vapor in the EP tube may accumulate as tiny droplets on the top, to avoid splashing when opening the lid, brief centrifugation over 2,000 $\mathrm{g}$ is necessary to centrifuge the possible droplets. At all times, keep the tube up-right to make sure that the CEES liquid is always in the bottom of the EP tube.

\subsection{Personal Protection}

\subsubsection{Eyes}

The eyes are commonly the most vulnerable in laboratory study and are very sensitive to vesicants $(10,11)$. Thus, equipment is essential for eye protection. The researcher must wear safety glasses in the lab to protect eyes from possible injury by the vapor and splash of the vesicant or any other hazardous chemical.

\subsubsection{Respiratory Tract}

Although a fume hood could effectively reduce vapor dispersion, a qualified gas mask is still necessary to protect the respiratory tract. In our study, we selected 3M gas masks (3301CN, China) as personal respiratory protection equipment. The central part of this mask is a filter filled with irregular-shape activated carbon for adsorbing the organic vapor.

\subsubsection{Hands}

CEES is known to be erosive to the skin. Our previous study proved that pits are quickly formed once the plastic plate contacts a CEES droplet (Figure 1). The gloves are made with different materials, including polyethylene (PE), polyvinyl chloride (PVC), latex, and nitrile rubber. Because of different anti-chemical abilities, gloves need to be selected according to application. Nitrile is a polyisoprene extract from petroleum. Compared to PE, PVC, and latex, nitrile has a better anti-chemical performance to grease, xylene, aliphatic solvent, and some pesticide formulas. In handling CEES, the researcher is recommended to wear nitrile gloves to protect hands. Butyl rubber gloves also have great anti-chemical performances and can be used in the experiment, even in handling SM. However, due to being heavy and inflexible, the researcher is not suggested to wear them when handling CEES (Table 1). For most studies, wearing two pairs of nitrile gloves is enough to protect hands when handling CEES.

\subsubsection{Others}

Since contamination with CEES in a lab is usually through respiratory inhalation or hand contact, heavy and inflexible anti-chemical clothing is not usually employed during the study. General laboratory clothing is enough for body protection, and the clothing collar and cuffs should be tightened before the study to avoid naked skin exposure. After each experiment, washing clothing can clean many chemicals attached to the surface. However, please do not reuse the clothing polluted by CEES, but change and discard it after decontamination. At the end of the experiment, the researcher should use neutral detergents to wash hands carefully no matter if any possible hazards were contacted during the experiment.

\subsection{Toxic Waste Management}

Some agents, such as chlorinated lime, chloramine T, and dibasic tricalcium hypochlorite, could oxidate or chlorinate the vesicants to make them less harmful. Thus, toxic wastes like culture media, pipette tips, EP tubes, and gloves should be decontaminated before discarding them.

\subsection{Decontamination}

Since no feeling early after CEES exposure, the researcher should be vigilant and take action once in contact. The principle of decontamination is to prevent the contacted tissues and cells from vesicant damage by physical removal or (and) chemical reaction. Timely decontamination is vital in preventing or reducing vesicant damage since most of SM on the skin would be absorbed within 10 min (12), and decontamination for eyes has weak efficiency over 2 min after vesicant exposure. The visible droplets should be first wiped using cotton swabs or paper towels. After that, use a large amount of clean water to flush the area exposed to vesicant. Eyes need to be flushed with water for 5 to 10 minutes. Chloramine T, a chemical commonly employed in flushing root canals at a concentration of $2 \%$, is highly efficient in vesicant decontamination and recommended to be used before water flushing. The chemical reactions are shown in Figure 2.

\subsection{Toxic Vapor Detection}

A normal person can smell SM in the air at a concentration as low as $0.07 \mathrm{mg} / \mathrm{m}^{3}$. However, because the vesicant could reduce the sensitivity of human smelling (13), the researchers may not be sensitive enough for the vesicant vapor. Routine detection in a lab is forcefully required for surveilling the leaked vapor. We have been equipped with portable GC-MS (Mars-400 Plus, Focused 

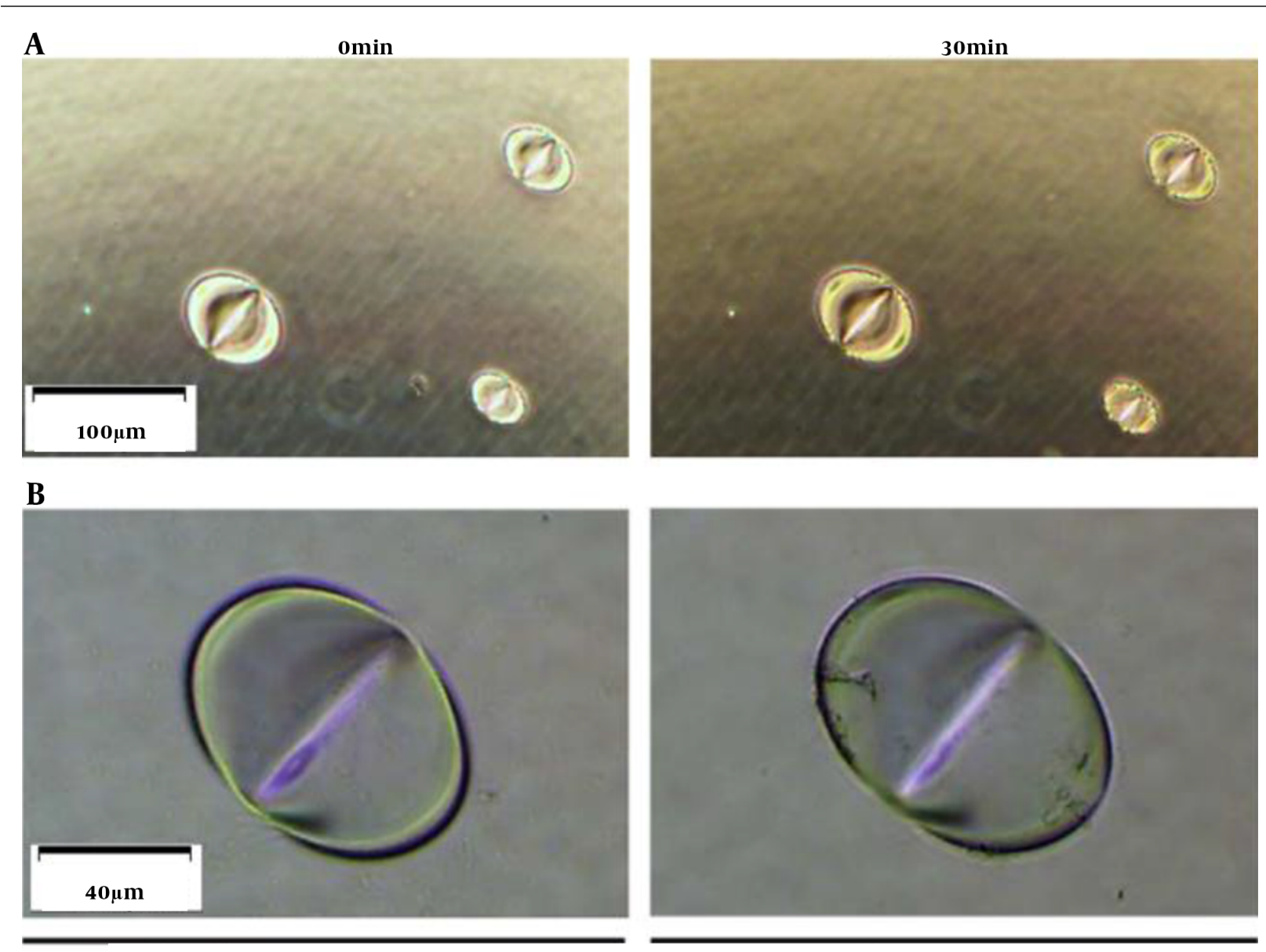

B

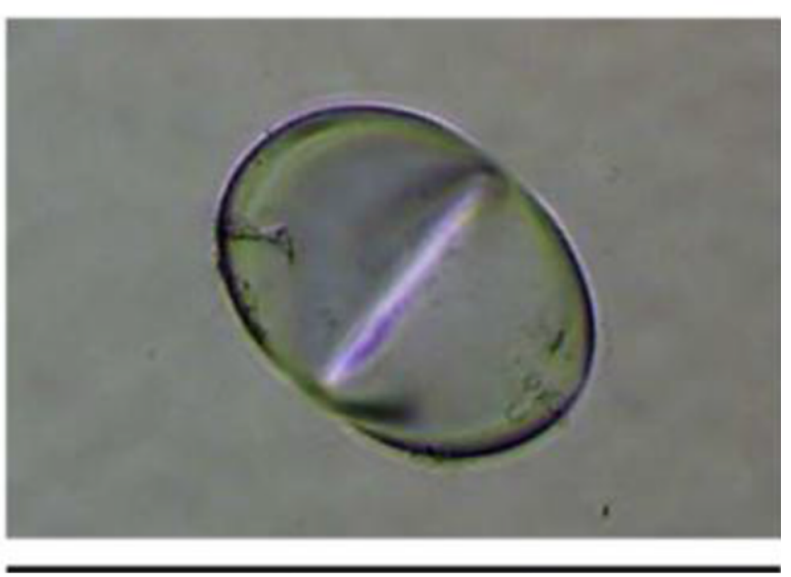

Before Scratch

After Scratch

Figure 1. Erosive characteristics of CEES. Under a microscope, (A) appearance change of plastic plate after contacting CEES droplets; (B) CEES droplet was scratched by a $1 \mathrm{~mL}$ tip. After scratching, the droplet shape did not change, and the erosive pit was confirmed.

\begin{tabular}{lcccc}
\hline Table 1. Comparison of Gloves with Different Materials ${ }^{\text {a }}$ & & & \\
\hline Variables & PE & PVC & Nitrile Rubber & Butyl Rubber \\
\hline Light & $\sqrt{ }$ & $\sqrt{ }$ & $\sqrt{ }$ \\
Flexible & $\sqrt{ }$ & $\sqrt{ }$ & $\sqrt{ }$ \\
CEES-resistant & - & - & $\sqrt{ }$ \\
\hline
\end{tabular}

a $\sqrt{ }$,yes or good; -, no or weak.

Photonics, Hangzhou, China) in our lab for years. The instrument works by analyzing the pattern of chemical fragment peaks in pumped air to identify any vesicant in the air. The device's detection sensitivity can reach the level of parts per billion (ppb, $1 \mu \mathrm{g} / \mathrm{m}^{3}$ ).

\section{Discussion}

Active sulfonium ions are formed once SM or CEES is dissolved in water $(14,15)$. They are unstable but highly toxic to cells as they can react with biomolecules like nucleotides and proteins. Both crosslinks (DNA-DNA or DNA-protein) and DNA/protein-adducts (CEES products) can be produced in SM-injured cells. Although less toxic than crosslinks, DNA/protein adducts are dominant in SM 
1.<smiles>CCSCC(Cl)Cl</smiles>

(CEES) (Chloramine T)

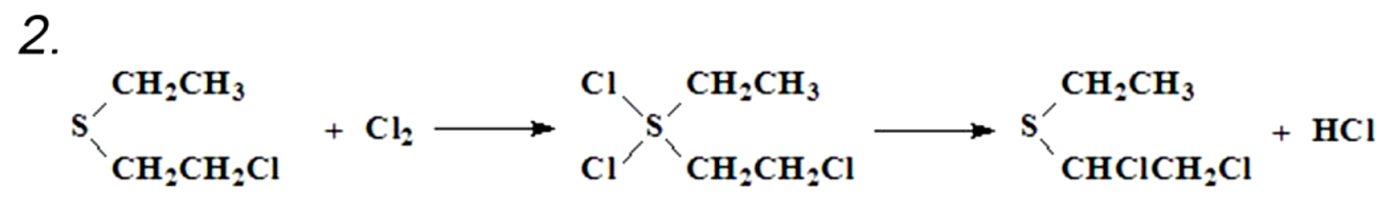

3.

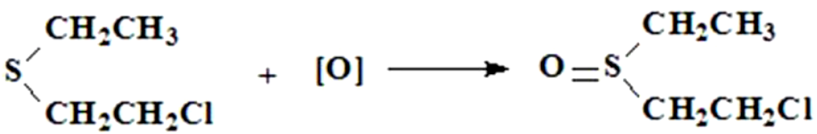

Figure 2. Chemical reactions between CEES and chloramine T. Note that nascent oxygen and $\mathrm{Cl}_{2}$ can be produced after chloramine $\mathrm{T}$ dissolves in water or ethanol, reacting with CEES.

products (about $80 \%$ ), and they may have long-term adverse effects on the poisoned cell $(16,17)$. Some known mechanisms are as follows: (1) CEES reduces RNA polymerase transcriptional activity to produce more RNA fragments of incomplete transcription (18); (2) only little CEESformed O6-adducts in the alkylated DNA are removed by O6-methylguanine-DNA methyltransferase (MGMT), which might cause cell mutation (19); (3) CEES inhibits cellular P450 enzyme system (20); and (4) CEES alkylates and inactivates many proteins/enzymes (21). In all, CEES is useful in the vesicant study for its less toxicity, but its chemical safety in laboratory study should be firstly and thoroughly educated to the new researcher.

\section{Footnotes}

Authors' Contribution: Feng Ye, conducted experimental study, designed the manuscript, drafted the MS, and made the final approval of the MS; Yan Sai, conducted administrative, technical, and logistic support; Zhongmin Zou, made revisions and discussions.

Conflict of Interests: The authors declare no conflicts of interest.

Funding/Support: The author(s) received no financial support for this article's research, authorship, and/or pub- lication.

\section{References}

1. Sezigen S, Ivelik K, Ortatatli M, Almacioglu M, Demirkasimoglu M, Eyison RK, et al. Victims of chemical terrorism, a family of four who were exposed to sulfur mustard. Toxicol Lett. 2019;303:9-15. doi: 10.1016/j.toxlet.2018.12.006. [PubMed:30572106].

2. Sezigen S, Kenar L. Recent sulfur mustard attacks in Middle East and experience of health professionals. Toxicol Lett. 2020;320:52-7. doi: 10.1016/j.toxlet.2019.12.001. [PubMed: 31805341].

3. Prescott T. Accidental sulfur mustard exposure from explosive ordnance in a UK military service person. BMJ Mil Health. 2021;167(4):2878. doi: 10.1136/bmjmilitary-2020-001687. [PubMed: 33243767].

4. IARC Working Group on the Evaluation of Carcinogenic Risks to Humans. Chemical agents and related occupations. Lyon (FR): International Agency for Research on Cancer; 2012.

5. Rancourt RC, Veress LA, Ahmad A, Hendry-Hofer TB, Rioux JS, Garlick RB, et al. Tissue factor pathway inhibitor prevents airway obstruction, respiratory failure and death due to sulfur mustard analog inhalation. Toxicol Appl Pharmacol. 2013;272(1):86-95. doi: 10.1016/j.taap.2013.05.020. [PubMed: 23727623]. [PubMed Central: PMC3775856].

6. Rancourt RC, Veress LA, Guo X, Jones TN, Hendry-Hofer TB, White CW. Airway tissue factor-dependent coagulation activity in response to sulfur mustard analog 2-chloroethyl ethyl sulfide. Am J Physiol Lung Cell Mol Physiol. 2012;302(1):L82-92. doi: 10.1152/ajplung.00306.2010. [PubMed: 21964405]. [PubMed Central: PMC3349374].

7. Tewari-Singh N, Jain AK, Inturi S, Agarwal C, White CW, Agarwal R. Silibinin attenuates sulfur mustard analog-induced skin injury by targeting multiple pathways connecting oxidative stress and inflamma- 
tion. PLoS One. 2012;7(9). e46149. doi: 10.1371/journal.pone.0046149. [PubMed: 23029417]. [PubMed Central: PMC3459894].

8. Jain AK, Tewari-Singh N, Gu M, Inturi S, White CW, Agarwal R. Sulfur mustard analog, 2-chloroethyl ethyl sulfide-induced skin injury involves DNA damage and induction of inflammatory mediators, in part via oxidative stress, in SKH-1 hairless mouse skin. Toxicol Lett. 2011;205(3):293-301. doi: 10.1016/j.toxlet.2011.06.019. [PubMed: 21722719]. [PubMed Central: PMC3207490].

9. O'Neil MJ, Smith A, Heckelman PE. The merck index: An encyclopedia of Chemicals. 14th ed. Whitehouse Station, NJ: Merck \& Co., Inc; 2006.342 p.

10. Ghasemi H, Owlia P, Ghazanfari T, Yaraee R, Saderi H, Soroush MR, et al. Conjunctival microbial florae in patients with seriously sulfur mustard induced eye injuries. Cutan Ocul Toxicol. 2013;32(1):13-7. doi: 10.3109/15569527.2012.692136. [PubMed: 22668347].

11. Ghasemi H, Mostafaie A, Yaraee R, Hassan ZM, Rezaei A, Mahmoudi $\mathrm{M}$, et al. Association of serum immunoglobulins levels and eye injuries in sulfur mustard exposed: Sardasht-Iran Cohort Study. Int Immunopharmacol. 2013;17(3):944-51. doi:10.1016/j.intimp.2012.12.027. [PubMed: 23370295].

12. Hejazi S, Soroush M, Moradi A, Khalilazar S, Mousavi B, Firooz A, et al. Skin manifestations in sulfur mustard exposed victims with ophthalmologic complications: Association between early and late phase. Toxicol Rep. 2016;3:679-84. doi: 10.1016/j.toxrep.2016.08.007. [PubMed: 28959592]. [PubMed Central: PMC5616011].

13. Veress LA, O'Neill HC, Hendry-Hofer TB, Loader JE, Rancourt RC, White $\mathrm{CW}$. Airway obstruction due to bronchial vascular injury after sulfur mustard analog inhalation. Am J Respir Crit Care Med.2010;182(11):135261. doi: 10.1164/rccm.200910-16180C. [PubMed: 20639443]. [PubMed Central: PMC3029928]

14. Xu B, Zong C, Nie Z, Guo L, Xie J. A novel approach for high sensitive determination of sulfur mustard by derivatization and isotope-dilution LC-MS/MS analysis. Talanta. 2015;132:245-51. doi: 10.1016/j.talanta.2014.09.015. [PubMed: 25476305].

15. Ye F, Dan G, Zeng Q, Chen M, Zhao Y, Cheng J, et al. Phospholipid and Triglyceride keep vortex-dispersed sulfur mustard analog as undissolved droplets which induce necrosis from nearby cells via declining mitochondrial membrane potential. Fundamental Toxicological Sciences. 2019;6(9):359-66. doi:10.2131/fts.6.359.

16. Jowsey PA, Williams FM, Blain PG. DNA damage responses in cells exposed to sulphur mustard. Toxicol Lett. 2012;209(1):1-10. doi: 10.1016/j.toxlet.2011.11.009. [PubMed: 22119920].

17. Sawyer TW, McNeely K, Louis K, Lecavalier P, Song Y, Villanueva M, et al. Comparative toxicity of mono- and bifunctional alkylating homologues of sulphur mustard in human skin keratinocytes. Toxicology. 2017;382:36-46. doi: 10.1016/j.tox.2017.03.005. [PubMed: 28285101].

18. Gray PJ, Phillips DR. Effect of alkylating agents on initiation and elongation of the lac UV5 promoter. Biochemistry. 1993;32(46):12471-7. doi: 10.1021/bio0097a027. [PubMed: 8241138].

19. Ludlum DB, Kent S, Mehta JR. Formation of O6-ethylthioethylguanine in DNA by reaction with the sulfur mustard, chloroethyl sulfide, and its apparent lack of repair by O6-alkylguanine-DNA alkyltransferase. Carcinogenesis.1986;7(7):1203-6. doi:10.1093/carcin/7.7.1203. [PubMed: 3719912].

20. Brimfield AA, Mancebo AM, Mason RP, Jiang JJ, Siraki AG, Novak MJ. Free radical production from the interaction of 2-chloroethyl vesicants (mustard gas) with pyridine nucleotide-driven flavoprotein electron transport systems. Toxicol Appl Pharmacol. 2009;234(1):12834. doi: 10.1016/j.taap.2008.10.002. [PubMed: 18977373]. [PubMed Central: PMC2703010].

21. Sayer NM, Whiting R, Green AC, Anderson K, Jenner J, Lindsay CD. Direct binding of sulfur mustard and chloroethyl ethyl sulphide to human cell membrane-associated proteins; implications for sulfur mustard pathology. J Chromatogr B Analyt Technol Biomed Life Sci. 2010;878(17-18):1426-32. doi: 10.1016/j.jchromb.2009.11.030. [PubMed: 20004628]. 